\title{
Versão Abreviada do WISC-III: Correlação entre QI Estimado e QI Total em Crianças Brasileiras ${ }^{1}$
}

\author{
Claudia Berlim de Mello \\ Nayara Argollo \\ Beatriz P. M. Shayer \\ Universidade Federal de São Paulo \\ Neander Abreu \\ Universidade Federal da Bahia \\ Kátya Godinho \\ Faculdade Ruy Barbosa \\ Paula Durán \\ Fernanda Vargem \\ Mauro Muszkat \\ Mônica Carolina Miranda \\ Orlando Francisco Amodeo Bueno \\ Universidade Federal de São Paulo
}

\begin{abstract}
RESUMO - Com o objetivo de correlacionar o QI Estimado com o QI Total, quatro bancos de dados do WISC III de 207 crianças foram associados: 1) crianças com desenvolvimento típico; 2) crianças com diagnóstico do transtorno do déficit de atenção e hiperatividade; 3 ) crianças referenciadas por dificuldades de aprendizagem em consultório particular; e 4) crianças com sequela neurológica avaliadas em ambulatório universitário. Os dados do QI total foram correlacionados aos do QI estimado, correspondentes à soma dos pontos ponderados dos subtestes Vocabulário e Cubos. Os resultados sugerem que o QI Estimado pode ser adotado quando há restrição de tempo e quando o desempenho intelectual está sendo usado como triagem em pesquisa, ou como ponto de referência dentro de uma avaliação neuropsicológica.
\end{abstract}

Palavras-chave: crianças; WISC III; QI estimado; QI Total

\section{Abbreviated Version of the WISC-III: Correlation between Estimated IQ and Global IQ of Brazilian Children}

\begin{abstract}
In order to calculate the correlation between the Estimated IQ and the Full Scale IQ of children submitted to the WISC III, four different data banks with a total of 207 children were integrated: (1) typically developing children, (2) children diagnosed as having Attention Deficit/ Hyperactivity Disorder, (3) children identified by private clinics as having learning disabilities (4) children with neurological sequelae diagnosed by a public university outpatient treatment program. Results suggest that the Estimated IQ, which is based on the weighted sum of the subtest scores of Cubes and Vocabulary, may be used in the presence of time constraints, when intellectual performance is important for screening in research procedures, and as a reference within a broader neuropsychological evaluation.
\end{abstract}

Keywords: Children; WISC III; Estimated IQ; Full Scale IQ.

A avaliação do desempenho intelectual global faz parte do diagnóstico psicológico na infância, constituindo um método ou técnica de uso privativo do psicólogo. No contexto multidisciplinar, a medida do coeficiente de inteligência é preconizada em manuais diagnósticos como o DSM-IV para a determinação e a classificação da deficiência intelectual e para o diagnóstico diferencial de transtornos do neurodesenvolvimento. A Escala Wechsler para crianças, disponível na sua terceira edição no Brasil (Wechsler, 2002) é amplamente utilizada para esta finalidade, sendo também útil na inves-

1 Os autores agradecem a AFIP - Fundo de Incentivo à Psicofarmacologia, pelo apoio financeiro e à direção acadêmica do curso de psicologia da Faculdade Ruy Barbosa pelo apoio logístico.

2 Endereço para correspondência: Centro Paulista de Neuropsicologia. Rua Embaú, 54. Vila Clementino. CEP: 04039-060. Fone: (11) 55498476. E-mail: cberlim@ib.usp.br tigação do perfil cognitivo em diversas condições clínicas, como no transtorno do déficit de atenção e hiperatividade (Mayes \& Calhoun, 2006), no autismo de alto funcionamento (Mayes \& Calhoun, 2008) e nos distúrbios de leitura e escrita (D’Angiulli \& Siegel, 2003).

O WISC III tem 13 subtestes, agrupados em uma escala verbal (Informação, Semelhanças, Aritmética, Vocabulário, Compreensão e Dígitos) e em uma escala de execução ou não-verbal (Completar Figuras, Arranjo de Figuras, Código, Cubos, Armar Objetos, Procurar Símbolos e Labirintos). A partir dos escores ponderados obtidos nos subtestes, chega-se aos Quocientes de Inteligência verbal (QIV) e de execução (QIE), além do Quociente global (QI). Além dos QIs, a escala provê índices fatoriais associados a competências especificas: Compreensão Verbal (abrangendo os subtestes Informação, Semelhanças, Vocabulário e Compreensão), Organização 
Perceptual (Completar Figuras, Arranjo de Figuras, e Armar Objetos), Resistência a Distração (Aritmética e Dígitos) e Velocidade de Processamento da Informação (Códigos e Procurar Símbolos).

O uso da escala WISC III é importante no acompanhamento de crianças com história de dificuldades de aprendizagem, uma vez que permite mensurar o desempenho intelectual global e separadamente dos domínios verbal e percepto-motor, bem como investigar especificamente as diferentes habilidades cognitivas expressas em cada subteste, delimitando as áreas cognitivas "fracas" e "fortes" da criança. Esta análise mais detalhada tem implicações nas intervenções educacionais e de reabilitação cognitiva.

A partir das publicações de Lezak (1995), as escalas de inteligência Wechsler ganharam também uma interpretação neuropsicológica, pela análise das habilidades subjacentes a cada subteste. A comparação entre o QIV e o QIE, por exemplo, pode render informações valiosas a respeito de disfunções inter-hemisféricas em casos de lesões cerebrais na infância, e se houver uma discrepância de mais de dez pontos entre eles, sugerir diagnósticos como os transtornos específicos de aprendizagem de leitura e escrita e o transtorno não-verbal de aprendizagem. A análise dos subtestes que compõem cada QI também contribui para a identificação de possibilidades diagnósticas em casos de transtornos neuropsiquiátricos na infância (Mayes \& Calhoun, 2004, Simões, 2002;).

A aplicação completa do WISC III é demorada, podendo ultrapassar duas horas, o que pode constituir um problema em situações clínicas nas quais o tempo disponível para a avaliação da criança é restrito. Situações desta natureza são comuns nos serviços públicos de atendimento de saúde, especialmente quando envolvem equipes multidisciplinares, onerando a família em gastos com transportes e ausências no trabalho, e em situações de pesquisa clínica, quando o instrumento é utilizado para triagem de nível intelectivo. Desse modo, formas reduzidas do WISC III tornariam os processos diagnósticos mais rápidos, disponibilizando tempo para a aplicação de outros instrumentos psicológicos e/ou neuropsicológicos, ou para outras consultas com a equipe multidisciplinar, além de diminuir custos nos dois cenários, com a mesma qualidade diagnóstica.

As formas reduzidas para obtenção do QI total de forma estimada se baseiam na aplicação de um menor número de subtestes, escolhidos pelas suas características psicométricas e correlação com o QI total, obtido pela aplicação de todos os subtestes do WISC. O número de subtestes aplicados varia entre dois (Vocabulário e Cubos) e quatro (Semelhanças, Vocabulário, Arranjo de Figuras, Cubos) (Simões, 2002), assim como os índices fatoriais (Compreensão Verbal, Organização Perceptual, Resistência à Distração e Velocidade de Processamento) (Donders, 2001). Outra versão reduzida das escalas Wechsler é a Wechsler Abbreviated Scale of Intelligence (WASI), que consiste em dois subtestes do domínio verbal (Vocabulário e Semelhanças) e dois subtestes do domínio de execução (Cubos e Matrizes de Raciocínio), gerando escores de QI verbal, QI de execução e QI total (Heck, Yates, Poggerelec, Tosi, Bandeira \& Trentini, 2009; Nascimento \& Figueiredo, 2002).
A aplicabilidade clínica de formas reduzidas do WISC III tem sido investigada já há alguns anos em casos de lesões cerebrais pediátricas por traumatismo crânio-encefálico (Donders, 1992; Donders \& Warschausky, 1996), nos transtornos de déficit de atenção e hiperatividade (Furgueson, McGuffin, Greenstein \& Soffer, 1998) e transtorno de conduta e oposicional desafiador (Furgueson, Greenstein, McGuffin \& Soffler, 1999), com correlações elevadas entre as formas reduzidas utilizando dois ou quatro subtestes (oscilando entre 0,87 a 0,97) e seis subtestes $(0,93$ a 0,95$)$ em comparação ao QI completo (Franzen \& Smith-Seemiller, 2001). Uma tabela que inclui QIs estimados com seus QIs globais correspondentes na forma constituída por apenas dois subtestes, Cubos e Vocabulário, é encontrada em Spreen e Strauss (1998).

Em nosso meio, vários serviços de neuropsicologia utilizam a forma reduzida de dois subtestes (Vocabulário e Cubos), a partir da tabela americana (Sattler,1992), como medida de triagem intelectual. Entretanto, não existem estudos de correlação na população brasileira entre o QI total obtido pela aplicação de toda a bateria do WISC III com aquele estimado pela tabela americana. O objetivo deste estudo foi correlacionar o QI total, obtido pela aplicação de todos os subtestes do WISC III, com o QI estimado utilizando-se a tabela americana de Sattler (1992), em grupos de crianças com desenvolvimento típico e em grupos clínicos.

\section{Método}

\section{Participantes}

A análise do presente estudo foi realizada em amostras de crianças entre seis e dezesseis anos de idade, de ambos os sexos, provenientes de escolas públicas e particulares das cidades de Salvador (Ba) e São Paulo(SP). Quatro bancos de dados com os resultados do WISC III completo foram associados. Estes bancos são provenientes de estudos prévios e que não tinham como objetivo serem representativos da população da cidade de origem. Como o objetivo deste estudo é correlacionar o QI Total com o Estimado na mesma amostra, a não representatividade da amostra não influencia nos resultados.

O primeiro banco de dados foi o primeiro estudo-piloto da normatização de um instrumento neuropsicológico (Argollo \& cols., 2009). Neste estudo, estudantes de psicologia previamente treinados aplicaram o WISC III em crianças com desenvolvimento típico de escolas públicas e particulares da cidade de Salvador.

O segundo banco de dados utilizado foi da tese de doutoramento (Abreu, 2007). Também neste estudo, o WISC III foi aplicado em dois grupos de crianças de escolas particulares da cidade de Salvador por estudantes de psicologia treinados: um grupo clínico (diagnóstico de transtorno do déficit de atenção e hiperatividade - TDAH, nos seus três subtipos, sem co-morbidades) e um grupo controle (desenvolvimento típico). 
O terceiro banco de dados utilizado foi de um ambulatório especializado em neuropsicologia infantil do Ambulatório de Neuroplasticidade do Núcleo de Atendimento em Neuropsicologia Infantil - NANI. Este ambulatório atende crianças com sequela neurológica de traumatismo craniano, neoplasia craniana, meningite, hemorragia intracraniana, doença vascular cerebral e má-formação cerebral. Neste serviço, o WISC III foi aplicado por psicólogas estagiárias ou pós-graduandas.

O quarto e último banco de dados utilizado foi de crianças atendidas em consultório particular de psicóloga especialista em dificuldades de aprendizagem. Para este consultório são referenciados casos de dificuldades de aprendizagem, tendo sido selecionadas aquelas com diagnóstico de Transtornos Específicos de Aprendizagem com ou sem TDAH.

Os dados, provenientes de contextos de atendimento - consultório particular, serviço público de diagnóstico em transtornos do neurodesenvolvimento e pesquisas realizadas em crianças com desenvolvimento típico e em grupos clínicos, abrangem uma amostra de crianças que são frequentemente submetidas a este exame. Os grupos clínicos incluíram crianças com transtorno do desenvolvimento, como o transtorno do déficit de atenção e hiperatividade, transtornos de aprendizagem e crianças com lesão cerebral adquirida, com amostras obtidas em serviços públicos e particulares, em comparação intergrupos. Este estudo não teve como objetivo comparar os grupos entre si (intra-grupos), uma vez que os bancos de dados são heterogêneos.

Ao final, o agrupamento das diversas amostras gerou um total de 207 sujeitos com WISC III completos e válidos, que no presente estudo foram nomeadas como Grupo A (sujeitos de desenvolviment típico), Grupo B (crianças com TDAH), Grupo C (crianças com história de dificuldades de aprendizagem associado ou não a TDAH) e Grupo D (crianças com lesão cerebral). A Tabela 1 resume as informações sobre os quatro grupos. Ressalta-se que as informações referentes à renda familiar e escolaridade dos pais não estavãodisponíveis para todos os grupos. No entanto, sendo o objetivo da presente análise realizar uma correlação intra-grupo, esse aspecto não afeta os resultados.

\section{Instrumento}

Foi utilizada na avaliação de todos os participantes a Escala Wechsler de Inteligência para Crianças 3a. Ed versão brasileira (Wechsler, 2002).

\section{Procedimento}

$\mathrm{Na}$ seleção das avaliações do WISC-III nos grupos relacionados ao ambulatório do serviço especializado e ao consultório particular foram considerados todos os casos atendidos nos últimos seis meses anteriores à análise. Os dados provenientes de consultório particular eram de uma das pesquisadoras participantes de coletas das pesquisas de uma das teses de doutoramento, utilizando a mesma metodologia de aplicação do instrumento. Nos demais grupos foi utilizado o banco de dados total fornecido pelos pesquisadores.

Em relação aos bancos de dados referentes a pesquisas (estudo de normatização e tese de doutoramento) e ao ambulatório do serviço especializado de atendimento a crianças com quadros neurológicos, foram identificados os termos de consentimento informado assinados pelos responsáveis. Em relação ao banco do consultório particular, o uso dos dados das crianças foi informado aos responsáveis, que assinaram termos de consentimento.

Para a análise estatística, os resultados do WISC III foram assciados em quatro grupos. O primeiro, Grupo A, foi constituído por crianças com o desenvolvimento típico provenientes dos estudos relacionados à normatização do instrumento e à tese de doutoramento. O segundo grupo (Grupo B) foi formado pelas amostra de crianças com TDAH considerando-se os seus três subtipos (desatento, hiperativo e combinado). O terceiro grupo (Grupo C) foi formado pela amostra de crianças avaliadas em conultório particular com queixa de dificuldade de aprendizagem com ou sem TDAH; O quarto e último grupo(Grupo D), foi constituído por crianças com lesão cerebral adquirida ou congênita.

Tabela 1. Características dos Bancos de Dados Utilizados no Estudo

\begin{tabular}{|c|c|c|c|c|c|c|c|c|}
\hline & \multirow{2}{*}{$\begin{array}{l}\text { Grupos } \\
\text { N (\%) } \\
\text { Desenv. } \\
\text { Típico }\end{array}$} & \multicolumn{2}{|c|}{ Grupo A } & \multicolumn{3}{|c|}{ Grupo B } & \multirow{2}{*}{$\begin{array}{c}\text { Grupo C } \\
\text { Lesão } \\
\text { Cerebral }\end{array}$} & \multirow[t]{2}{*}{ Grupo D } \\
\hline & & $\begin{array}{l}\text { Desenv. } \\
\text { Típico }\end{array}$ & $\begin{array}{c}\text { TDAH } \\
\text { (Desatento) }\end{array}$ & $\begin{array}{c}\text { TDAH } \\
\text { (Hiperativo) }\end{array}$ & $\begin{array}{c}\text { TDAH } \\
\text { (Combinado) }\end{array}$ & $\begin{array}{l}\text { Dif. Aprend/ } \\
\text { TDAH }\end{array}$ & & \\
\hline $\mathbf{N}$ & (\% do total) & $61(29,47)$ & $43(20,77)$ & $17(8,21)$ & $11(5,31)$ & $16(7,73)$ & $44(21,26)$ & $15(7,24)$ \\
\hline \multirow[t]{2}{*}{ Idade } & $\mathrm{M} \pm \mathrm{DP}$ & $8,97 \pm 1,96$ & $12,21 \pm 1,07$ & $12,94 \pm 1,01$ & $12,40 \pm 1,36$ & $12,97 \pm 1,20$ & $10,40 \pm 2,82$ & $10,55 \pm 2,86$ \\
\hline & Md (min.--máx.) & $9,0(6-12)$ & $13,4(11-15)$ & $13(11-14)$ & $11,8(11-15)$ & $13,3(11-15)$ & $10(8-15)$ & $9,96(6-16)$ \\
\hline Sexo & Masculino & $27(44,3)$ & $12(27,9)$ & $15(88,2)$ & $10(90,9)$ & $10(62,5)$ & $28(63,6)$ & $8(53,3)$ \\
\hline \multirow[t]{2}{*}{ Escola } & Pública & $31(50,8)$ & $0(0,0)$ & $0(0,0)$ & $0(0,0)$ & $0(0,0)$ & $0(0,0)$ & $15(100)$ \\
\hline & Particular & $30(49,2)$ & $43(100)$ & $17(100)$ & $11(100)$ & $11(100)$ & $44(100)$ & $0(0,0)$ \\
\hline
\end{tabular}


Tabela 2. Escores do QI Estimado (Spreen \& Strauss, 1998)

\begin{tabular}{cccccccc}
\hline $\mathbf{C}+\mathbf{V}$ & QI Estimado & $\mathbf{C}+\mathbf{V}$ & QI Estimado & $\mathbf{C}+\mathbf{V}$ & QI Estimado & $\mathbf{C}+\mathbf{V}$ & QI Estimado \\
\hline 1 & 45 & 11 & 74 & 21 & 103 & 31 & 132 \\
2 & 48 & 12 & 77 & 22 & 106 & 32 & 135 \\
3 & 51 & 13 & 80 & 23 & 109 & 33 & 138 \\
4 & 54 & 14 & 83 & 24 & 112 & 34 & 141 \\
5 & 56 & 15 & 85 & 25 & 115 & 35 & 144 \\
6 & 59 & 16 & 88 & 26 & 117 & 36 & 146 \\
7 & 62 & 17 & 91 & 27 & 120 & 37 & 149 \\
8 & 65 & 18 & 94 & 28 & 123 & 38 & 152 \\
9 & 68 & 19 & 97 & 29 & 126 & 39 & 155 \\
10 & 71 & 20 & 100 & 30 & 129 & 40 & 158 \\
\hline
\end{tabular}

Legenda: CV: Cubos + Vocabulário; QIE: QI Estimado. Extraído de Sattler (1992).

Dos bancos de dados, foram coletados o resultado do QI total, calculado pela aplicação da bateria WISC III completa, e os resultados dos escores ponderados dos subtestes Cubos e Vocabulário. Os escores destes dois subtestes foram somados e confrontados com a tabela americana modificada de Sattler (Spreen \& Strauss, 1998) para o cálculo do QI Estimado (Tabela 2).

A hipótese do estudo é que o QI estimado se correlaciona com o QI total e o nível de significância de 1\% foi escolhido para diminuir o erro tipo alfa. O Teste Paramétrico de Pearson foi usado para correlacionar o QI estimado e o QI total, seguindo a interpretação da Tabela 3.

Tabela 3. Interpretação do valor de $\pi$ (rho)

\begin{tabular}{cc}
\hline Valor do $\boldsymbol{\pi}$ & Interpretação \\
\hline 0,00 a 0,19 & Correlação bem fraca \\
\hline 0,20 a 0,39 & Correlação fraca \\
\hline 0,40 a 0,69 & Correlação moderada \\
\hline 0,70 a 0,89 & Correlação forte \\
\hline 0,90 a 1,00 & Correlação muito forte \\
\hline
\end{tabular}

\section{Resultados e Discussão}

Informações sobre as características demográficas da amostra total e das amostras constituídas pelo grupo de crianças com desenvolvimento típico e pelos grupos clínicos encontram-se descritas na Tabela 4. Como o objetivo deste estudo foi a comparação pareada na mesma amostra (o QI Total e o QI Estimado na mesma criança-amostra), esta não precisava, necessariamente, ser representativa da população da qual derivou. Portanto, é importante enfatizar que o valor do QI Total não representa a média das populações das cidades participantes. Como pode ser observado, o QI médio encontrado neste estudo é superior à média descrita para a população brasileira. Esta amostra inclui pais de escolaridade e nível socioeconômico alto, o que explica uma média mais alta do QI total (Ceci \& Williams, 1997).

A Tabela 4 revela que as amostras compostas por crianças com desenvolvimento típico e com diagnósticos neuropsiquiátricos são caracterizadas por faixas etárias semelhantes, e que em relação ao sexo dos participantes a frequência de meninos em comparação à de meninas foi menor no grupo típico e maior no grupo com as patologias, em termos percentuais. Quanto às variáveis socioeconômicas, considerando-se a amostra total, a maior parte das crianças era oriunda de famílias com pais com alta escolaridade (acima de 16 anos de estudo), com renda familiar mais distribuída entre as faixas de menos de cinco até 20 salários mínimos (SM). Nos dois grupos, a renda é mais concentrada na faixa a partir dos 11 SM, embora uma aparente diferença em termos percentuais seja observada na faixa dos 11 a $20 \mathrm{SM}$, mais alta no grupo com desenvolvimento típico.

Conforme descrito na Tabela 5, quando se comparam as características demográficas nos diferentes grupos clínicos separadamente, verifica-se uma maior variação de faixa etária nas crianças com dificuldades de aprendizagem (de seis a 13 anos e três meses) em comparação aos demais diagnósticos. O maior número de sujeitos do sexo masculino nas amostras (com exceção do grupo com TDAH combinado) parece refletir dados de estudos epidemiológicos que apontam maior frequência deste sexo nos transtornos do neurodesenvolvimento. Cabe ressaltar, em relação a essas variáveis de níveis sócio-econômicos relacionadas à escolaridade dos pais, as diferenças dos bancos de dados quanto à disponibilidade dessas informações, o que sugere uma falta de uniformidade metodológica entre as diversas modalidades de atendimento que incluem avaliações de desempenho intelectual em casos suspeitos de transtornos do neurodesenvolvimento.

As correlações entre o QI estimado e o QI total dos subgrupos estudados encontram-se na Tabela 6. Os resultados revelaram correlações muito elevadas entre o QI estimado, obtido na forma reduzida do WISC III baseada nos subtestes Cubos e Vocabulário, e o QI global aferido pela escala com- 
Tabela 4. Características Demográficas da Amostra

\begin{tabular}{|c|c|c|c|c|}
\hline \multicolumn{2}{|c|}{ Grupos } & Amostra & Crianças Típicas & $\begin{array}{c}\text { Patologia Neuropsiqui- } \\
\text { átrica }\end{array}$ \\
\hline $\mathbf{N}$ & $(\%)$ & $207(100)$ & $104(50,24)$ & $103(49,76)$ \\
\hline Idade & $\mathrm{M} \pm \mathrm{DP}$ & $11,11 \pm 2,63$ & $10,72 \pm 2,66$ & $11,50 \pm 2,54$ \\
\hline \multicolumn{2}{|c|}{ Mediana (mínimo - máximo) } & $11,50(6,00-16,30)$ & $11,07(6,00-15,08)$ & $11,80(6,01-16,30)$ \\
\hline Sexo & Masculino & $110(53,1)$ & $39(37,5)$ & $71(68,9)$ \\
\hline Escola & Particular & $161(77,8)$ & $73(70,2)$ & $88(85,4)$ \\
\hline \multirow{5}{*}{ Escolar. Pai ${ }^{1}$} & $<4$ & $10(4,8)$ & $9(8,7)$ & $1(1,0)$ \\
\hline & $4 a<8$ & $13(6,3)$ & $11(10,6)$ & $2(1,9)$ \\
\hline & $8 \mathrm{a}<11$ & $7(3,4)$ & $5(4,8)$ & $2(1,9)$ \\
\hline & $11 \mathrm{a}<16$ & $30(14,5)$ & $14(13,5)$ & $16(15,5)$ \\
\hline & $\geq 16$ & $83(40,1)$ & $60(57,7)$ & $23(22,3)$ \\
\hline \multirow{4}{*}{ Escolar. Mãe ${ }^{2}$} & $<4$ & $13(6,3)$ & $13(12,5)$ & $0(0,0)$ \\
\hline & $4 a<8$ & $10(4,8)$ & $10(9,6)$ & $0(0,0)$ \\
\hline & $8 \mathrm{a}<11$ & $7(3,4)$ & $5(4,8)$ & $2(1,9)$ \\
\hline & $11 \mathrm{a}<16$ & $24(11,6)$ & $13(12,5)$ & $11(10,7)$ \\
\hline \multirow{5}{*}{$\begin{array}{c}\text { Renda } \\
\text { Familiar }^{3}\end{array}$} & $\geq 16$ & $91(44,0)$ & $62(59,6)$ & $29(28,2)$ \\
\hline & $<5$ & $37(17,9)$ & $31(29,8)$ & $6(5,8)$ \\
\hline & $5-10$ & $23(11,1)$ & $5(4,8)$ & $18(17,5)$ \\
\hline & $11-20$ & $34(16,4)$ & $28(26,8)$ & $6(5,8)$ \\
\hline & $>20$ & $1(0,5)$ & $37(35,6)$ & $43(41,7)$ \\
\hline
\end{tabular}

1. Em 64 (30,9\%) questionários não havia descrição da escolaridade do pai; 2. Em 62 (30,0\%) não havia descrição da escolaridade da mãe; 3 . Em 64 (30,4\%) crianças não havia informações sobre a renda familiar. Escolaridade dos pais em anos de estudos e renda familiar em salários mínimos.

pleta. Tais correlações se revelaram muito fortes no grupo de crianças com desenvolvimento típico e fortes no grupo formado por casos de Transtorno do Déficit de Atenção. Estas observações indicam que esta forma reduzida pode ser usada em contexto clinico, envolvendo processos de diagnóstico nosológico e diferencial nestes transtornos. Por outro lado, nos casos de lesões cerebrais verificou-se a presença de uma correlação apenas moderada, o que leva a considerar que este nestes casos o uso de formas reduzidas não é o mais adequado. Novos estudos são indicados para a verificação da aplicabilidade destes procedimentos em outros transtornos do neurodesenvolvimento. Cabe ressaltar o QI médio elevado entre as crianças com desenvolvimento típico, o que pode refletir maior nível de escolaridade e renda familiar nesta amostra. $\mathrm{O}$ estudo não teve como objetivo descrever a média de QI da população brasileira, uma vez que não se trata de amostra representativa da população brasileira. Limitações com o uso de banco de dados de estudos previamente realizados não inviabiliza os resultados, porque as comparações foram intra-grupos (na mesma criança), entretanto dados referentes à escolaridade e renda das crianças provenientes do ambulatório e consultório particular não puderam ser analisados.

Em suma, as análises de correlação obtidas deste estudo indicam que a forma reduzida do WISC III, baseada nos subtestes Cubos e Vocabulário, equivale à forma global em condições pediátricas não associadas a lesões cerebrais. Cabe considerar, entretanto, que o QI estimado não deve substituir a medida do QI global baseado em todos os subtestes em situações nas quais há maior necessidade de planejamento de programas de intervenção educacional ou em reabilitação cognitiva. Seu uso deve ser adotado em condições específicas em que há restrição de tempo e em que a investigação do desempenho intelectual consiste apenas em mais uma de diferentes avaliações necessárias para uma melhor definição diagnóstica. Ou seja, a adequação da forma reduzida deve ser analisada quando o fator tempo é um dos mais relevantes para a eficiência da intervenção.

Os resultados obtidos neste estudo devem ser considerados em termos de uma tentativa inicial de investigação das 
Tabela 5. Características das Amostras dos Grupos Clínicos

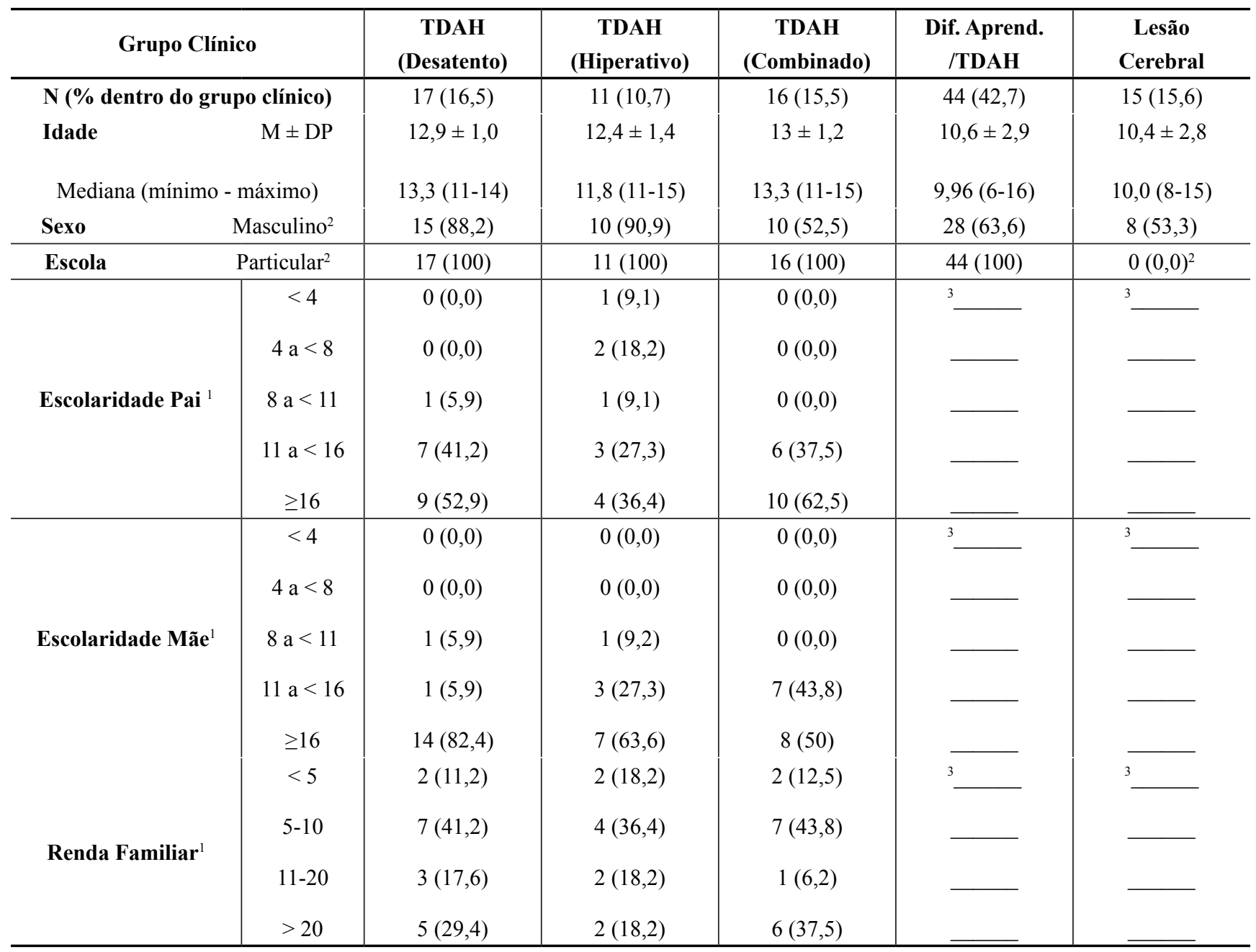

1. Percentual dentro do grupo; 2. 15 (100\%) de escola pública; 3. Dados não disponíveis. Escolaridade dos pais em anos de estudos e renda familiar em salários mínimos.

Tabela 6. Correlação entre QI Total e QI Estimado - Significância a 1\%

\begin{tabular}{|c|c|c|c|c|c|c|}
\hline \multirow{2}{*}{ Grupos } & \multirow{2}{*}{ N (\%) } & \multirow{2}{*}{$\begin{array}{l}\text { QI Total } \\
M \pm \mathbf{D P}\end{array}$} & \multirow{2}{*}{$\begin{array}{c}\text { QI Estimado } \\
\mathbf{M} \pm \mathbf{D P}\end{array}$} & \multicolumn{2}{|c|}{$\begin{array}{l}\text { Correlação de } \\
\text { Pearson }\end{array}$} & \multirow{2}{*}{ Valor do $p$} \\
\hline & & & & Valor & Interpretação & \\
\hline Desenvolvimento Típico & $104(50,2)$ & $111 \pm 21$ & $111 \pm 18$ & 0,91 & Muito forte & 0,000000 \\
\hline TDAH (Desatento) & $17(8,2)$ & $102 \pm 13$ & $102 \pm 12$ & 0,88 & Forte & 0,000003 \\
\hline TDAH (Hiperativo) & $11(5,3)$ & $109 \pm 14$ & $112 \pm 18$ & 0,85 & Forte & 0,000995 \\
\hline TDAH (Combinado) & $16(7,7)$ & $107 \pm 14$ & $112 \pm 13$ & 0,83 & Forte & 0,000073 \\
\hline DA/TDAH & $44(21,3)$ & $104 \pm 14$ & $107 \pm 15$ & 0,81 & Forte & 0,000000 \\
\hline Lesão Cerebral & $15(7,2)$ & $84 \pm 16$ & $90 \pm 10$ & 0,60 & Moderada & 0,018001 \\
\hline Amostra Total & $207(100)$ & $107 \pm 19$ & $108 \pm 17$ & 0,88 & Forte & 0,000000 \\
\hline
\end{tabular}

QI estimado: baseado na soma dos escores ponderados em Cubos e Vocabulário. 
possibilidades do uso da versão abreviada do WISC-III baseada em dois subtestes em diferentes contextos de atendimento (clinico e de pesquisa). A obtenção de uma amostragem com base em banco de dados pré-existentes limita analises mais amplas na comparação inter grupos. Novos estudos são necessários para a investigação adequada da propriedade do uso de versão abreviadas do WISC-III e mesmo na validade dos escores da tabela de Sattler.

\section{Referências}

Abreu, J. N. S. (2007). Memória e Transtorno do Déficit de Atenção/ Hiperatividade. Tese de Doutorado, Universidade de São Paulo, São Paulo.

Argollo, N., Bueno, O.F.A., Shayer, B., Godinho, K., Abreu, K., Duran, P., Assis, A., Lima, F., Silva, T., Guimarães, J., Carvalho, R., Moura, I., \& Seabra, A.G. (2009). Adaptação transcultural da bateria NEPSY: Avaliação neuropsicológica do desenvolvimento. Avaliação Psicológica, 8(1), 59-75.

Ceci, S. J., \& Williams, W. M. (1997). Schooling, intelligence and income. American Psychologist, 52, 1051-1058.

D'Angiulli, A., \& Siegel, L. S. (2003). Cognitive functioning as measured by the WISC-R: Do children with learning disabilities have distinctive patterns of performance? Journal of Learning Disabilities, 36(1), 48-58.

Donders, J. (1992). Validity of two short forms of the WISC-R in children with traumatic brain injury. Journal of Clinical Psychology, 48(3):364-370.

Donders, J. (2001). Using a short form of the WISC III: Sinful or smart? Child Neuropsychology, 7(2), 99-103.

Donders, J., \& Warschausky, S. (1996). Validity of a short form of the WISC III in children with traumatic head injury. Child Neuropsychology, 2,227-232.

Franzen, M. D., \& Smith-Seemiller, L. (2001). Comparison among seven different short forms of the WISC III. [Resumo] Archives of Clinical Neuropsychology, 16, 825.

Furgueson, C., Greenstein, D., McGuffin, P., \& Soffer, S. (1999). Efficacy of the WISC III short form for children diagnosed with conduct and oppositional defiant disorders [Resumo]. Archives of Clinical Neuropsychology, 14, 6-7.

Furgueson, C., McGuffin, P., Greenstein, D., \& Soffer, S. (1998). Efficacy of the WISC III short form for children diagnosed with ADHD [Resumo]. Archives of Clinical Neuropsychology, 13,10 .
Heck, V. S., Yates, D. B., Poggere, L. C., Tosi, S. P., Bandeira, D. R., \& Trentini, C. V. (2009). Validação dos subtestes verbais da versão de adaptação da WASI. Avaliação Psicológica, 8, 33-42.

Lezak, M. (1995). Neuropsychological assessment. New York: Oxford University Press.

Mayes, S. D., \& Calhoun, S. L. (2004). Similarities and differences in Wechsler Intelligence Scale for Children - Third Edition (WISC III) profiles: Support for subtest analysis in clinical referrals. Clinical Neuropsychologist, 18(4), 559-572.

Mayes, S. D., \& Calhoun, S. L. (2006). WISC-IV and WISCIII profiles in children with ADHD. Journal of Attention Disorders, 9(3), 486-493.

Mayes, S. D., \& Calhoun, S. L. (2008). WISC-IV and WIAT-II profiles in children with high-functioning autism. Journal of Autism and Developmental Disorders, 38(3), 428-439.

Nascimento, E. \& Figueiredo, V. L. M. (2002). WISC III e WAIS-III: Alterações nas versões originais americanas decorrentes das adaptações para uso no Brasil. Psicologia: Reflexão e Crítica, 15(3), 603-612.

Sattler, J. (1992). Assessment of children: WISC-II and WWPSI-R supplement. San Diego: Jerome M. Sattler Publisher.

Simões, M. R. (2002). Utilizações da WISC-III na avaliação neuropsicológica de crianças e adolescentes. Paidéia, 12, 113-132.

Spreen, O. \& Strauss, E. (1998). A Compendium of Neuropsychological Tests. Administration, Norms and Commentary (2a ed.). New York: Oxford University Press.

Wechsler, D. (2002). Escala de Inteligência Wechsler para Crianças ( $3^{\text {a }}$ ed.): Manual; Adaptação e padronização de uma amostra brasileira, $1^{a}$ ed; V. L. M. Figueiredo. São Paulo: Casa do Psicólogo (trabalho original publicado em 1991).

\author{
Recebido em 03.02.2009 \\ Primeira decisão editorial em 10.05.2010 \\ Versão final em 30.12.2010 \\ Aceito em 30.12.2010
}

